\title{
Effect of the Patient-Centered Medical Home on Racial Disparities in Quality of Care
}

\author{
Karen E. Swietek, PhD MPH ${ }^{1,2}$, Bradley N. Gaynes, MD MPH ${ }^{3}$, \\ George L. Jackson, PhD MHA ${ }^{4,5,6}$, Morris Weinberger, $P h D^{7}$, and Marisa Elena Domino, \\ $P h D^{1,7}$
}

\begin{abstract}
'Department of Health Policy and Management, Gillings School of Global Public Health, University of North Carolina at Chapel Hill, Chapel Hill, NC, USA; ${ }^{2}$ NORC at the University of Chicago, Chicago, IL, USA; ${ }^{3}$ Department of Psychiatry, UNC School of Medicine, Chapel Hill, NC, USA; ${ }^{4} \mathrm{Center}$ of Innovation to Accelerate Discovery and Practice Transformation (ADAPT) Durham Veterans Affairs Health Care System, Durham, NC, USA; ${ }^{5}$ Department of Population Health Sciences, Duke University, Durham, NC, USA; ${ }^{6}$ Division of General Internal Medicine, Duke University, Durham, NC, USA; ${ }^{7}$ Cecil G. Sheps Center for Health Services Research, Chapel Hill, NC, USA.
\end{abstract}

\begin{abstract}
BACKGROUND: Research demonstrates that the patientcentered medical home (PCMH) is associated with improved clinical outcomes and quality of care, and the populations that can most benefit from this model require long-term management, e.g., persons with chronic illness and behavioral health conditions. However, different populations may not benefit equally from the $\mathrm{PCMH}$, and empirical evidence about the effects of this model on racial disparities is limited.
\end{abstract}

OBJECTIVE: Estimate the association between enrollment in National Committee for Quality Assurance (NCQA)-recognized PCMHs and racial disparities in quality of care for adults with major depressive disorder (MDD) and comorbid medical conditions.

DESIGN: Applying a quasi-experimental instrumental variable design to account for differential selection into the $\mathrm{PCMH}$, we used generalized estimating equations to determine the probability of receiving eight diseasespecific quality measures.

SUBJECTS: Medicaid enrollees in three states not dually enrolled in Medicare, ages 18-64 with MDD and > 1 other chronic condition. A subgroup analysis was conducted for enrollees with comorbid diabetes.

INTERVENTIONS: Enrollment in an NCQA-recognized PCMH.

MAIN MEASURES: Disease-specific quality indicators for MDD (e.g., antidepressant use, receipt of psychotherapy), and for diabetes, (e.g. Alc testing, LDL-C testing, retinal exams, and medical attention for nephropathy).

KEY RESULTS: PCMH enrollment was associated with an increase in the overall likelihood of receiving six of eight recommended services and a decrease in the likelihood of receiving any psychotherapy (4.94 percentage points, $p<0.01)$ and retinal exams (5.51 percentage points, $p<0.05)$. Although both groups improved, PCMH enrollment was associated with an exacerbation of the Blackwhite disparity in adequate antidepressant use by 4.20 percentage points $(p<0.01)$.

CONCLUSIONS: While PCMH enrollment may improve the overall quality of care, the effect is inconsistent across

Received August 30, 2019

Revised December 23, 2019

Accepted February 10, 2020

Published online February 24, 2020 racial groups and not always associated with reductions in racial disparities in quality.

KEY WORDS: patient-centered medical homes; racial disparities; quality of care; chronic conditions.

$\mathrm{J}$ Gen Intern Med 35(8):2304-13

DOI: $10.1007 / \mathrm{s} 11606-020-05729-\mathrm{x}$

(c) Society of General Internal Medicine 2020

\section{BACKGROUND}

Persons with comorbid physical and behavioral health conditions are a priority for the U.S. healthcare system. Mental illnesses are the most prevalent comorbidities among highcost Medicaid enrollees, and this population utilizes more healthcare across a wider range of services than the general population, making coordination of their care more difficult and leading to adverse health outcomes, higher mortality, and greater spending. ${ }^{1-4}$ Because behavioral health conditions complicate the management of physical disorders, ${ }^{5}$ coordinating care for persons with co-occurring conditions like major depressive disorder (MDD) may contribute to suboptimal quality of care. ${ }^{3,6}$

The intersection between physical and behavioral chronic conditions may be especially complex for racial minorities, who are disproportionately at risk for chronic comorbidities. ${ }^{1}$ Compared with non-Hispanic-whites with common psychiatric disorders, Black and Hispanic/Latino patients with similar conditions report higher rates of diabetes, obesity, and cardiovascular disease. ${ }^{1}$ Receipt of high-quality care for chronic conditions differs across racial/ethnic groups among people with mental illness. ${ }^{7}$ Given that racial/ethnic minorities are more likely to seek mental health services from primary care than mental health specialists, ${ }^{8}$ understanding the potential for primary care models to address the intersection between race, MDD, and chronic disease is critical to reduce disparities. ${ }^{1,9}$, 10

In primary care, the patient-centered medical home $(\mathrm{PCMH})$ has the potential to improve clinical outcomes in 
complex populations with chronic physical and behavioral health conditions by organizing care delivery around a primary care provider (PCP) who leads a team responsible for coordinating patients' overall healthcare needs. ${ }^{11-16}$ Several organizations certify PCMHs; the most widely recognized program was developed by the National Committee on Quality Assurance (NCQA) in $2008^{17}$ and has been endorsed by a broad coalition of stakeholders, including national health plans, patient groups, and every major national physician organization. ${ }^{18,} 19$ Adoption of the PCMH has expanded rapidly; in 2018, 29 states reported serving some portion of their Medicaid population through a PCMH. ${ }^{20,21}$

However, different populations may not benefit equally from this model. Evidence about the effects of the PCMH in vulnerable populations is limited, and studies of racial disparities in quality in PCMH initiatives report mixed results. ${ }^{13,22-}$

${ }^{24}$ One study found the PCMH reduced or eliminated racial disparities in preventive care reminders, cholesterol testing, and cancer screenings; ${ }^{25}$ others observed varying effects among racial/ethnic groups, ${ }^{19},{ }^{26-28}$ with some finding no effect. ${ }^{29}$ Because the PCMH is being widely adopted in Medicaid, assessing its potential to reduce disparities has significant policy implications. ${ }^{20,}{ }^{21}$ We investigated the effects of NCQA-recognized PCMH practices on racial disparities in quality among Medicaid enrollees with comorbid MDD and chronic physical conditions.

\section{METHODS}

\section{Setting}

We include 2008-2011 Medicaid claims from three states with relatively high rates of Medicaid enrollment and complete claims data that were available for re-use for this analysis: North Carolina, Georgia, and Texas. This study was exempted from review by the Office of Human Research Ethics at the University of North Carolina at Chapel Hill (Study no. 162551).

\section{Sample}

We linked several administrative data sources: claims from the 2008-2011 Medicaid Analytic eXtract (MAX), provider specialty from the National Plan and Provider Enumeration System (NPPES), and PCMH recognition status from NCQA. We measured county-level supply of mental health professionals using data from the Area Health Resource File (AHRF) ${ }^{30}$ and county-level socioeconomic status (SES) using the U.S. Census Bureau's Small Area Income and Poverty Estimates. ${ }^{31}$ The study population includes Medicaid enrollees age 1864 years with MDD and > 1 other chronic medical condition. Dual enrollees in Medicare and Medicaid were excluded due to potentially incomplete pharmacy and utilization data.

We conducted subgroup analyses for enrollees with diabetes because of its high prevalence in Medicaid populations and potential to interfere with managing MDD; diabetes also serves as a key tracer condition for understanding chronic care delivery more generally. ${ }^{4}{ }^{32}$ Further, racial/ethnic minorities experience higher rates of both conditions, and comorbid depression and diabetes is predictive of suboptimal outcomes in these groups. ${ }^{32}$

To avoid "rule-out" diagnoses and/or coding errors, we required $>1$ inpatient diagnosis or $>2$ outpatient or emergency department diagnoses of MDD or diabetes during a single year in the study period, and $>1$ claim for the condition in each year. We excluded individuals with serious mental illnesses (e.g., schizophrenia, bipolar disorder) because they are likely to see a behavioral health specialist as their primary point of care. $^{33}$

\section{Measures}

Disease-specific quality indicators for MDD or diabetes derived from Medicaid claims (Appendix Table 7) were chosen from recommended core quality measures for the $\mathrm{PCMH},{ }^{34}$ the Centers for Medicare and Medicaid Services 2016 core set of adult quality measures for Medicaid, ${ }^{35}$ and the Healthcare Effectiveness Data and Information Set (HEDIS). ${ }^{36}$ To track treatment modality, we measured the likelihood that enrollees received any psychotherapy or antidepressant prescription; either modality is considered guideline-concordant for MDD. ${ }^{37,38}$

Seven recommended services were measured annually. Given the episodic nature of MDD, we also assessed the HEDIS antidepressant management measure for acute-phase treatment, defined as the likelihood that enrollees received > 84 days of antidepressants during an acute (120-day) episode. ${ }^{37,}, 39$ Initiation of an acute episode was defined as 1) two outpatient services for MDD on different dates or 2) initiation of an antidepressant prescription. We required a washout period of $>3$ months of Medicaid enrollment without MDD claims before a new episode. The end of an acute phase was defined as either 90 days without receiving MDD services or antidepressants or 120 days after the episode started. ${ }^{37}$

The exposure of interest was enrollment in an NCQAcertified PCMH. Enrollees were attributed to the PCP (defined using taxonomy codes for internal medicine, family medicine, pediatrics, general practice, ambulatory clinics or centers, nurse practitioners, or physician assistants) that delivered the plurality of their non-hospital evaluation and management visits. ${ }^{40}$ A provider's PCMH status was determined using NCQA recognition data. Enrollees receiving the same number of services from multiple providers were attributed to the provider with the most recent service date. ${ }^{40}$ We conducted sensitivity analyses defining PCMH enrollment as having any claim with an NCQA-recognized PCMH provider during a given year rather than using the modal provider's status.

Enrollee-level covariates included age, sex, number of additional chronic comorbidities (defined using the Healthcare Cost and Utilization Project Chronic Condition Indicator), ${ }^{41}$ 
number of Medicaid-enrolled months, and rurality. Providerlevel covariates included Federally Qualified Health Center or Rural Health Center status, and sex. We measured the supply of mental health professionals using county availability of psychiatrists and county-level Mental Health Professional Shortage Area (HPSA) status (full or partial). Finally, county-level SES measures included percent of the population under poverty and median income.

New enrollment in a PCMH can take one of two forms: seeking care from a recognized PCMH by switching providers or staying with a provider who became a PCMH. In either case, enrollment in a PCMH is non-random. To address possible selection bias from patient- or provider-level factors, we constructed two instrumental variables based on county rates of PCMH adoption: (1) the overall ratio of county PCMH adoption, defined as the number of PCMH providers in a county divided by the number of PCPs as measured by NCQA PCMH recognition data and the AHRF and (2) a similar county-level rate of NCQA medical home practices to all PCPs, contingent on the provider NPI appearing in Medicaid MAX claims during the study period. Both instruments are hypothesized to be associated with participation in a PCMH, regardless of the mechanism. Regional variation is assumed to be otherwise independent of enrollee-level quality outcomes, meaning that the only reason a region with a higher penetration of PCMH practices would have higher quality levels is through individual and practice participation decisions.

\section{Defining and Measuring Disparities}

The Institute of Medicine (IOM) defines a racial disparity as "racial or ethnic differences in the quality of health care that are not due to access-related factors or clinical needs, preferences, and appropriateness of intervention." ${ }^{42}$ This definition distinguishes between the simple unadjusted difference in means or rates between racial groups and disparities driven by discrimination or other systematic factors. ${ }^{42,}{ }^{43}$ Further, it includes the effects of mediating factors (other than health status and preferences), such as geography or SES. ${ }^{42}$

Implementing the IOM definition to measure disparities requires generating counterfactual predictions of outcomes that minority groups would experience if their health status were identical to their white counterparts, adjusting for health status but no other factors explaining differences in quality or utilization. ${ }^{43}$ In a nonlinear model, estimating this counterfactual requires simulation. ${ }^{43-45}$ Notably, this estimation method differs from analyses estimating a coefficient in a multivariate regression model while adjusting for other covariates. This estimator, the "residual direct effect" of race/ethnicity, is not concordant with the IOM definition of a disparity in that it does not distinguish between variable which may be legitimate drivers of differences in utilization and mediating factors such as SES which may represent race-related disadvantages. ${ }^{45}$

Therefore, to estimate disparities consistent with the IOM definition we followed a process developed by McGuire and colleagues: using non-Hispanic-whites as a reference group, we first fit a model describing relationships between quality/ utilization and health status, race, and other characteristics. We then transformed the distributions of health status variables (e.g., age, gender, number of comorbidities) from the minority groups to be the same as those of non-Hispanic-whites, while leaving other variables unchanged; this created a counterfactual minority subgroup with distributions identical to the white subgroup. We then generated predictions using coefficients from the initial models and the transformed/counterfactual health status variables for minority groups by PCMH status, and aggregated predictions by racial group. To estimate precision, we generated measures of statistical significance using bootstrapping. ${ }^{43}$

\section{Statistical Analysis}

We used generalized estimating equations (GEE) with a binomial family and logit link function. We selected an independent correlation structure selected based on the quasilikelihood under the independence model criterion (QIC). ${ }^{46}$ To address differential selection into the PCMH, we used twostage residual inclusion (2SRI), an instrumental variable technique. Average marginal effects for the overall estimates were obtained using predictive margins. In the disparity models, predicted probabilities were obtained using the methods described above. All analyses were conducted using Stata (version 14).

The instruments were uniformly strong, with F-statistics of the joint significance of the two instruments ranging from 1048.92 to $4029.4{ }^{47}$ The correlation between the instruments was 0.44 , suggesting that although the two measure similar constructs, they capture different information and are both appropriate for inclusion in these models. The results of IV models should be interpreted as local average treatment effects, specifically the effect of the PCMH on outcomes for those who enroll because of county infrastructure or level of interest in medical homes. ${ }^{48}$

\section{RESULTS}

The sample (Table 1) consisted of 310,906 person-years contributed by 191,565 unique individuals; $1.6 \%$ of person-years were accounted for by PCMH enrollees. PCMH enrollees were more likely to be Black $(39.2 \%$ versus $30.1 \%$, $p<0.001)$ and less likely to live in rural areas $(\mathrm{p}<0.001)$ than non-enrollees. In unadjusted comparisons (Table 2), PCMH enrollees were significantly $(p<0.01)$ more likely to receive all recommended services except for psychotherapy and retinal exams.

\section{Major Depressive Disorder Outcomes}

PCMH enrollment was associated with a 4.88 percentage point increase in the overall receipt of any MDD treatment, 
Table 1 Summary Statistics (Person-Years)

\begin{tabular}{|c|c|c|c|}
\hline & \multicolumn{3}{|c|}{$N(\%)$ or mean(SD) } \\
\hline & Total & Non-PCMH & РСМН \\
\hline$N$ & 310,906 & $306,040(98.40 \%)$ & $4866(1.60 \%)$ \\
\hline Diabetes & $82,501(26.50 \%)$ & $81,304(26.60 \%)$ & $1197(24.60 \%)$ \\
\hline \multicolumn{4}{|l|}{ Race/ethnicity } \\
\hline White & $156,039(50.20 \%)$ & $153,529(50.20 \%)$ & $2510(51.60 \%)$ \\
\hline Black & $94,167(30.30 \%)$ & $92,259(30.10 \%)$ & $1908(39.20 \%)$ \\
\hline Hispanic/Latino & $37,516(12.10 \%)$ & $37,329(12.20 \%)$ & $187(3.80 \%)$ \\
\hline Female enrollee & $246,694(79.30 \%)$ & $242,765(79.30 \%)$ & $3929(80.70 \%)$ \\
\hline Age & $40.82(13.57)$ & $40.83(13.57)$ & $40.15(13.35)$ \\
\hline Months of Medicaid enrollment & $10.771(2.40)$ & $10.77(2.40)$ & $10.801(2.32)$ \\
\hline Chronic comorbidities & $5.23(4.31)$ & $5.23(4.31)$ & $5.32(4.26)$ \\
\hline County $\%$ under poverty & $18.80(5.72)$ & $18.81(5.75)$ & $18.16(3.63)$ \\
\hline County median income & $43,726.33$ & $43,702.24$ & $45,240.96$ \\
\hline & (9316.43) & (9351.32) & $(6595.7111)$ \\
\hline \multicolumn{4}{|l|}{ Rurality } \\
\hline Metro-adjacent & $65,664(21.10 \%)$ & $65,280(21.30 \%)$ & $384(7.90 \%)$ \\
\hline Rural & $17,363(5.60 \%)$ & $17,292(5.70 \%)$ & $71(1.50 \%)$ \\
\hline \multicolumn{4}{|c|}{ Mental Health Professional Shortage Area (HPSA) } \\
\hline Whole county & $120,531(38.80 \%)$ & $119,977(39.20 \%)$ & $554(11.40 \%)$ \\
\hline Partial county & $113,380(36.50 \%)$ & $110,821(36.20 \%)$ & $2559(52.60 \%)$ \\
\hline County supply of psychiatrists & $49.70(89.47)$ & $49.43(89.45)$ & $66.47(88.62)$ \\
\hline \multicolumn{4}{|l|}{ State } \\
\hline GA & $84,958(27.30 \%)$ & $84,661(27.70 \%)$ & $297(6.10 \%)$ \\
\hline $\mathrm{NC}$ & $137,198(44.10 \%)$ & $132,995(43.50 \%)$ & $4203(86.40 \%)$ \\
\hline TX & $88,750(28.50 \%)$ & $88,384(28.90 \%)$ & $366(7.50 \%)$ \\
\hline \multicolumn{4}{|l|}{ Year } \\
\hline 2008 & $63,713(20.50 \%)$ & $63,713(20.80 \%)$ & $0(0.0 \%)$ \\
\hline 2009 & $83,764(26.90 \%)$ & $83,674(27.30 \%)$ & $90(1.80 \%)$ \\
\hline 2010 & $94,296(30.30 \%)$ & $92,649(30.30 \%)$ & $1647(33.80 \%)$ \\
\hline 2011 & $69,133(22.20 \%)$ & $66,004(21.60 \%)$ & $3129(64.30 \%)$ \\
\hline
\end{tabular}

whether psychotherapy or antidepressants, among enrollees with MDD $(p<0.01)$ (Table 3). This improvement was driven primarily by antidepressant use; PCMH enrollment was associated with a 5.94 percentage point higher rate of receipt of any antidepressants $(p<0.01)$, but also with a 4.94 percentage point overall lower rates of psychotherapy $(p<0.01)$. In the acute episodic MDD analyses, PCMH enrollment was associated with a 2.85 percentage point higher probability of receiving minimally adequate antidepressants $(p<0.05)$.

In the disparities models (Table 4), Black and Hispanic/ Latino enrollees had higher predicted probabilities of receiving annual psychotherapy than white enrollees in both the non-PCMH and PCMH groups $(50.9 \%$ and $41.1 \%$ respectively, compared with $39.6 \%$ for whites); this pattern persisted among PCMH enrollees. However, this effect was heterogeneous across racial groups: PCMH enrollees had a lower predicted probability of receiving any psychotherapy for white and Black enrollees and a small increase for Hispanic/Latino enrollees. PCMH enrollment was associated with significant reductions in Black-white and Hispanic-white disparities in calendar-year antidepressant use $(0.90$ percentage points, $p<0.05$ and 9.72 percentage points, $p<0.01$, respectively). PCMH enrollment was also associated with a 6.81 percentage point reduction in

Table 2 Unadjusted Rates of Quality Metrics

\begin{tabular}{|c|c|c|c|}
\hline & Non-PCMH $N(\%)$ & PCMH $N(\%)$ & $p$ value ( $t$ test) \\
\hline \multicolumn{4}{|l|}{ MDD } \\
\hline \multicolumn{4}{|l|}{ Calendar-year } \\
\hline Any psychotherapy & $109,510(40.6 \%)$ & $1721(39.3 \%)$ & 0.066 \\
\hline Any antidepressants & $159,948(59.4 \%)$ & $3174(72.4 \%)$ & $<0.001$ \\
\hline Psychotherapy or antidepressants & $203,058(75.4 \%)$ & $3728(85.1 \%)$ & $<0.001$ \\
\hline$N$ & 269,460 & 4383 & \\
\hline \multicolumn{4}{|l|}{ Acute episode } \\
\hline Min. antidepressants & $40,297(24.0 \%)$ & $1035(33.6 \%)$ & $<0.001$ \\
\hline$N$ & 167,910 & 3076 & \\
\hline \multicolumn{4}{|l|}{ Diabetes } \\
\hline A1c testing & $52,963(65.0 \%)$ & $918(76.7 \%)$ & $<0.001$ \\
\hline Retinal exam & $26,780(32.9 \%)$ & $346(28.9 \%)$ & 0.004 \\
\hline Lipid panel & $40,450(49.7 \%)$ & $751(62.8 \%)$ & $<0.001$ \\
\hline Attn for nephropathy & $24,151(29.6 \%)$ & $489(40.9 \%)$ & $<0.001$ \\
\hline$N$ & 81.483 & 1197 & \\
\hline
\end{tabular}


Table 3 Average Marginal Effect of PCMH Enrollment on MDD Outcomes

\begin{tabular}{|c|c|c|c|c|}
\hline & \multicolumn{3}{|l|}{ Calendar-year } & \multirow{2}{*}{$\begin{array}{l}\text { Acute episode } \\
\text { Adequate antidepressants }\end{array}$} \\
\hline & Any psychotherapy & Any antidepressants & $\begin{array}{l}\text { Psychotherapy or } \\
\text { antidepressants }\end{array}$ & \\
\hline PCMH enrollment & $\begin{array}{l}-0.050 * * \\
(0.011)\end{array}$ & $\begin{array}{l}0.059 * * \\
(0.011)\end{array}$ & $\begin{array}{l}0.048 * * \\
(0.0099)\end{array}$ & $\begin{array}{l}0.029 * \\
(0.012)\end{array}$ \\
\hline \multicolumn{5}{|l|}{ Race } \\
\hline Black & $\begin{array}{l}0.033 * * \\
(0.0030)\end{array}$ & $\begin{array}{l}-0.14 * * \\
(0.0029)\end{array}$ & $\begin{array}{l}-0.074 * * \\
(0.0026)\end{array}$ & $\begin{array}{l}-0.15 * * \\
(0.0025)\end{array}$ \\
\hline Hispanic/Latino & $\begin{array}{l}0.037 * * \\
(0.0046)\end{array}$ & $\begin{array}{l}-0.055 * * \\
(0.0044)\end{array}$ & $\begin{array}{l}-0.022 * * \\
(0.0035)\end{array}$ & $\begin{array}{l}-0.080 * * \\
(0.0048)\end{array}$ \\
\hline Chronic Conditions & $\begin{array}{l}-0.0049 * * \\
(0.00029)\end{array}$ & $\begin{array}{l}0.0094 * * \\
(0.00028)\end{array}$ & $\begin{array}{l}0.00099 * * \\
(0.00024)\end{array}$ & $\begin{array}{l}0.0078 * * \\
(0.00030)\end{array}$ \\
\hline \multicolumn{5}{|l|}{ Rurality } \\
\hline Non-metro (adjacent to urban area) & $\begin{array}{l}-0.00014 \\
(0.0031)\end{array}$ & $\begin{array}{l}-0.010 * * \\
(0.0030)\end{array}$ & $\begin{array}{l}-0.0080 * * \\
(0.0027)\end{array}$ & $\begin{array}{l}-0.0095 * * \\
(0.032)\end{array}$ \\
\hline Non-metro (non-urban adjacent) & $\begin{array}{l}-0.040 * * \\
(0.0050)\end{array}$ & $\begin{array}{l}-0.019 * * \\
(0.0049)\end{array}$ & $\begin{array}{l}-0.036 * * \\
(0.0045)\end{array}$ & $\begin{array}{l}-0.00094 \\
(0.0052)\end{array}$ \\
\hline Enrollee age & $\begin{array}{l}-0.0037 * * \\
(8.58 \mathrm{e}-05)\end{array}$ & $\begin{array}{l}-0.0016^{* *} \\
(8.53 \mathrm{e}-05)\end{array}$ & $\begin{array}{l}-0.0034 * * \\
(7.65 \mathrm{e}-05)\end{array}$ & $\begin{array}{l}0.002-* * \\
(8.84 \mathrm{e}-05)\end{array}$ \\
\hline Female enrollee & $\begin{array}{l}0.0093 * * \\
(0.0026)\end{array}$ & $\begin{array}{l}0.059 * * \\
(0.0025)\end{array}$ & $\begin{array}{l}0.040 * * \\
(0.0023)\end{array}$ & $\begin{array}{l}0.033 * * \\
(0.0027)\end{array}$ \\
\hline Months of Medicaid enrollment & $0.017 * *(0.00046)$ & $0.011 * *(0.00042)$ & $\begin{array}{l}0.015 * * \\
(0.00035)\end{array}$ & $\begin{array}{l}0.0093 * * \\
(0.00052)\end{array}$ \\
\hline FQHC & $\begin{array}{l}-0.011 * * \\
(0.0038)\end{array}$ & $\begin{array}{l}0.053 * * \\
(0.0036)\end{array}$ & $\begin{array}{l}0.033 * * \\
(0.0031)\end{array}$ & $\begin{array}{l}0.0083 * \\
(0.0041)\end{array}$ \\
\hline $\mathrm{RHC}$ & $\begin{array}{l}-0.028 * * \\
(0.0048)\end{array}$ & $\begin{array}{l}0.036 * * \\
(0.0045)\end{array}$ & $\begin{array}{l}0.022 * * \\
(0.0038)\end{array}$ & $\begin{array}{l}0.030 * * \\
(0.0051)\end{array}$ \\
\hline Female provider & $\begin{array}{l}0.0053 * \\
(0.0026)\end{array}$ & $\begin{array}{l}0.044 * * \\
(0.0025)\end{array}$ & $\begin{array}{l}0.034 * * \\
(0.0021)\end{array}$ & $\begin{array}{l}0.024 * * \\
(0.0027)\end{array}$ \\
\hline County percent under poverty & $0.0016 * *(0.00036)$ & $-0.0039 * *(0.00035)$ & $-0.0012 * *(0.00030)$ & $-0.0033 * *(0.00040)$ \\
\hline County median income & $\begin{array}{l}1.62 \mathrm{e}-06 * * \\
(2.33 \mathrm{e}-07)\end{array}$ & $\begin{array}{l}-1.79 \mathrm{e}-06 * * \\
(2.24 \mathrm{e}-07)\end{array}$ & $\begin{array}{l}-2.96 \mathrm{e}-07 \\
(1.96 \mathrm{e}-07)\end{array}$ & $\begin{array}{l}-9.14 \mathrm{e}-07 * * \\
(2.50 \mathrm{e}-07)\end{array}$ \\
\hline County no. of psychiatrists & $0.00018 * *(1.51 \mathrm{e}-05)$ & $8.87 \mathrm{e}-05 * *(1.41 \mathrm{e}-05)$ & $\begin{array}{l}0.00013 * * \\
(1.16 \mathrm{e}-05)\end{array}$ & $\begin{array}{l}-1.65 \mathrm{e}-05 \\
(1.79 \mathrm{e}-05)\end{array}$ \\
\hline Mental health HPSA & & & & \\
\hline Whole county & $\begin{array}{l}0.014 * * \\
(0.0031)\end{array}$ & $\begin{array}{l}-0.0039 \\
(0.0030)\end{array}$ & $\begin{array}{l}-0.00084 \\
(0.0027)\end{array}$ & $\begin{array}{l}0.00048 \\
(0.0032)\end{array}$ \\
\hline Partial county & $\begin{array}{l}0.023 * * \\
(0.0028)\end{array}$ & $\begin{array}{l}-0.019 * * \\
(0.0028)\end{array}$ & $\begin{array}{l}-0.0018 \\
(0.0025)\end{array}$ & $\begin{array}{l}-0.0082^{* * *} \\
(0.0029)\end{array}$ \\
\hline State & & & & \\
\hline North Carolina & $\begin{array}{l}-0.026 * * \\
(0.0030) \\
-0.16^{* *} \\
(0.0033)\end{array}$ & $\begin{array}{l}0.078 * * \\
(0.0028) \\
-0.16 * * \\
(0.0035)\end{array}$ & $\begin{array}{l}0.042 * * \\
(0.0023) \\
-0.16 * * \\
(0.0032)\end{array}$ & $\begin{array}{l}0.036 * * \\
(0.0032) \\
-0.077 * * \\
(0.0035)\end{array}$ \\
\hline Year & & & & \\
\hline 2010 & $\begin{array}{l}0.0015 \\
(0.0025)\end{array}$ & $\begin{array}{l}-0.025^{* *} \\
(0.0024)\end{array}$ & $\begin{array}{l}-0.012 * * \\
(0.0021)\end{array}$ & $\begin{array}{l}-0.011 * * \\
(0.0026)\end{array}$ \\
\hline 2011 & $\begin{array}{l}-0.0015 \\
(0.0028)\end{array}$ & $\begin{array}{l}-0.0020 \\
(0.0028)\end{array}$ & $\begin{array}{l}0.0021 \\
(0.0025)\end{array}$ & $\begin{array}{l}0.036 * * \\
(0.0031)\end{array}$ \\
\hline Pearson residual & $\begin{array}{l}-0.049 * * \\
(0.0098)\end{array}$ & $\begin{array}{l}0.059 * * \\
(0.010)\end{array}$ & $\begin{array}{l}0.049 * * \\
(0.0087)\end{array}$ & $\begin{array}{l}0.0019 \\
(0.0023)\end{array}$ \\
\hline
\end{tabular}

Bootstrapped standard errors in parentheses

$* * p<0.01$, * $p<0.05$

Hispanic-white disparities $(p<0.01)$ in receipt of either calendar-year measure. Finally, in episodic MDD analyses, PCMH enrollment was associated with a 4.20 percentage point increase in Black-white disparities $(p<0.01)$, but not with Hispanic-white disparities.

\section{Diabetes Outcomes}

Among 82,501enrollees with diabetes (Table 5), PCMH enrollment was generally associated with significant $(p<0.01)$ increases in overall receipt of the overall probability of receiving a lipid panel (11.70 percentage points), A1c test (14.0 percentage points), and attention for nephropathy ( 7.53 percentage points). PCMH enrollment was associated with a 5.51 percentage point decrease in the likelihood of receiving a retinal exam $(p<0.05)$.

In disparity models (Table 6), Black enrollees not enrolled in a PCMH were 2.27 percentage points less likely to receive A1c testing $(p<0.01)$; there was no statistically significant disparity among PCMH enrollees. Statistically significant Black-white disparities also existed for both receipt of lipid testing and retinal exams. PCMH enrollment was associated with a non-significant reduction in Black-white disparities in lipid testing (from 7.36 to 3.71 percentage points) and retinal exams (from 6.45 to 5.70 percentage points). There were no statistically significant Black-white disparities in attention for nephropathy. Hispanic/Latino enrollees had higher rates of A1c testing and this advantage persisted among PCMH 
Table 4 Comparison of Predicted Probabilities and Disparities Changes for MDD Outcomes

\begin{tabular}{|c|c|c|c|c|c|}
\hline Any psychotherapy (annual) & White & Black & Disparity & Hispanic/Latino & Disparity \\
\hline Non-PCMH & $\begin{array}{l}0.40 * * \\
(0.0017)\end{array}$ & $\begin{array}{l}0.51 * * \\
(0.0025)\end{array}$ & $\begin{array}{l}-0.11 * * \\
(0.0030)\end{array}$ & $\begin{array}{l}0.41 * * \\
(0.0035)\end{array}$ & $\begin{array}{l}-0.015 * * \\
(0.0040)\end{array}$ \\
\hline PCMH & $0.37 * *$ & $\begin{array}{l}0.47 * * \\
(0.0083)\end{array}$ & $\begin{array}{l}-0.096^{* *} \\
(0.0042)\end{array}$ & $\begin{array}{l}0.45^{* *} \\
(0.011)\end{array}$ & $\begin{array}{l}-0.077 * * \\
(0.0084)\end{array}$ \\
\hline$\Delta$ Disparity & & & $\begin{array}{l}0.017^{* * *} \\
(0.0032)\end{array}$ & & $\begin{array}{l}-0.062 * * \\
(0.0074)\end{array}$ \\
\hline \multicolumn{6}{|l|}{ Any antidepressants (annual) } \\
\hline Non-PCMH & $\begin{array}{l}0.67 * * \\
(0.0016)\end{array}$ & $\begin{array}{l}0.53^{* *} \\
(0.0026)\end{array}$ & $\begin{array}{l}0.14 * * \\
(0.0031)\end{array}$ & $\begin{array}{l}0.44 * * \\
(0.0039)\end{array}$ & $\begin{array}{l}0.22 * * \\
(0.0042)\end{array}$ \\
\hline РCMH & $\begin{array}{l}0.78^{* *} \\
(0.0064)\end{array}$ & $\begin{array}{l}0.65^{* *} \\
(0.0082)\end{array}$ & $\begin{array}{l}0.13 * * \\
(0.0046)\end{array}$ & $\begin{array}{l}0.66^{* *} \\
(0.012)\end{array}$ & $\begin{array}{l}0.13 * * \\
(0.010)\end{array}$ \\
\hline$\Delta$ Disparity & & & $\begin{array}{l}-0.0090 * \\
(0.0038)\end{array}$ & & $\begin{array}{l}-0.097 * * \\
(0.0094)\end{array}$ \\
\hline \multicolumn{6}{|c|}{ Psychotherapy or antidepressants (annual) } \\
\hline Non-PCMH & $\begin{array}{l}0.79 * * \\
(0.0014)\end{array}$ & $\begin{array}{l}0.77 * * \\
(0.0021)\end{array}$ & $\begin{array}{l}0.018 * * \\
(0.0025)\end{array}$ & $\begin{array}{l}0.69 * * \\
(0.0033)\end{array}$ & $\begin{array}{l}0.099 * * \\
(0.0036)\end{array}$ \\
\hline PCMH & $\begin{array}{l}0.87 * * \\
(0.0050)\end{array}$ & $\begin{array}{l}0.85^{* *} \\
(0.0058)\end{array}$ & $\begin{array}{l}0.022 * * \\
(0.0033)\end{array}$ & $\begin{array}{l}0.84 * * \\
(0.0087)\end{array}$ & $\begin{array}{l}0.031 * * \\
(0.0072)\end{array}$ \\
\hline$\Delta$ Disparity & & & $\begin{array}{l}0.0040 \\
(0.0030)\end{array}$ & & $\begin{array}{l}-0.068 * * \\
(0.0071)\end{array}$ \\
\hline \multicolumn{6}{|c|}{ Adequate antidepressants (episodic) } \\
\hline Non-PCMH & $\begin{array}{l}0.27 * * \\
(0.0017)\end{array}$ & $\begin{array}{l}0.12 * * \\
(0.0015)\end{array}$ & $\begin{array}{l}0.15^{* *} \\
(0.0022)\end{array}$ & $\begin{array}{l}0.11 * * \\
(0.0021)\end{array}$ & $\begin{array}{l}0.20 * * \\
(0.0027)\end{array}$ \\
\hline PCMH & $\begin{array}{l}0.38 * * \\
(0.0094)\end{array}$ & $\begin{array}{l}0.18^{* *} \\
(0.0061)\end{array}$ & $\begin{array}{l}0.20 * * \\
(0.0054)\end{array}$ & $\begin{array}{l}0.20 * * \\
(0.011)\end{array}$ & $\begin{array}{l}0.18 * * \\
(0.0092)\end{array}$ \\
\hline$\Delta$ Disparity & & & $\begin{array}{l}0.042 * * \\
(0.0050)\end{array}$ & & $\begin{array}{l}0.0076 \\
(0.0085)\end{array}$ \\
\hline
\end{tabular}

Bootstrapped Standard Errors in Parentheses

$* * p<0.01, * p<0.05$

enrollees. There were no statistically significant Hispanicwhite disparities for either treatment group in receipt of lipid panels or attention for nephropathy. A statistically significant Hispanic-white disparity existed in the PCMH group for retinal exams, but not for the non-PCMH group, suggesting that disparities were exacerbated among PCMH enrollees.

\section{Sensitivity Analyses}

Sensitivity analyses defining PCMH attribution as any claim with an NCQA-recognized provider produced results similar to the primary analyses in direction and significance, though the effect sizes were generally smaller in magnitude. One exception was the annual receipt of psychotherapy, where the effect of PCMH enrollment was almost double the primary analyses.

\section{DISCUSSION}

PCMH enrollment was associated with an increased likelihood of receiving six of eight recommended services; this is consistent with literature suggesting that the PCMH can improve the quality of care in Medicaid populations. ${ }^{15,} 49$ The two exceptions to this finding, psychotherapy and retinal exams, may result from these services not typically being provided in primary care settings. However, PCMH enrollment was associated with an increase in the receipt of any MDD treatment.

Some providers and policymakers believe that the PCMH should improve outcomes for all racial groups, with minority groups benefitting more because they have the most room for improvement. ${ }^{23}$ Overall, our findings do not support this belief; we only see this pattern in Black-white disparities in any antidepressant use and Alc testing and Hispanic-white disparities in any antidepressant use and use of either psychotherapy or antidepressants. In other cases (e.g., Black-white disparities in adequate episodic antidepressant use), PCMH enrollment improves outcomes for both the white and minority groups, but the disparity between the two is slightly exacerbated.

Notably, we found that minority enrollees (especially Hispanic/Latinos) were more likely to receive several recommended services than their white counterparts. These advantages generally persisted between the non-PCMH and PCMH groups. One possible explanation is that this sample is limited to individuals enrolled in Medicaid; previous studies have found that Hispanic-white disparities are largely driven by disparities in insurance coverage. ${ }^{50,51}$ Another possibility is that we do not control for English fluency or language concordance between enrollee and provider. Previous studies have found that among insured enrollees, English-speaking Hispanic/Latino enrollees show a healthcare use pattern similar to non-Hispanic-white enrollees, while Spanish-speaking Hispanic/Latino enrollees had a significantly lower likelihood of having a physician visit or visit with a mental health provider, or receiving preventive services. ${ }^{52}$

Many disparity-related concerns raised by our findings are reflected in the NCQA's updated 2017 PCMH recognition standards. These new standards have several criteria relating to equity of care, including "targeting population health 
Table 5 Average Marginal Effect of PCMH Enrollment on Diabetes Outcomes

\begin{tabular}{|c|c|c|c|c|}
\hline & Lipids & A1c & Retinal Exam & Attn for Nephropathy \\
\hline PCMH Enrollment & $\begin{array}{l}0.12 * * \\
(0.022)\end{array}$ & $\begin{array}{l}0.14 * * \\
(0.020)\end{array}$ & $\begin{array}{l}-0.055^{*} \\
(0.025)\end{array}$ & $\begin{array}{l}0.075 * * \\
(0.026)\end{array}$ \\
\hline \multicolumn{5}{|l|}{ Race } \\
\hline Black & $\begin{array}{l}-0.020 * * \\
(0.0048)\end{array}$ & $\begin{array}{l}0.0060 \\
(0.0053)\end{array}$ & $\begin{array}{l}0.022 * * \\
(0.0045)\end{array}$ & $\begin{array}{l}0.034 * * \\
(0.0051)\end{array}$ \\
\hline Hispanic/Latino & $\begin{array}{l}0.034 * * \\
(0.0073)\end{array}$ & $\begin{array}{l}0.048 * * \\
(0.0083)\end{array}$ & $\begin{array}{l}0.045 * * \\
(0.0073)\end{array}$ & $\begin{array}{l}0.069 * * \\
(0.0074)\end{array}$ \\
\hline Chronic Conditions & $\begin{array}{l}0.0068 * * \\
-0.00038\end{array}$ & $\begin{array}{l}0.010 * * \\
(-0.00041)\end{array}$ & $\begin{array}{l}0.015^{*} * \\
-0.00037\end{array}$ & $\begin{array}{l}0.013 * * \\
-0.00036\end{array}$ \\
\hline \multicolumn{5}{|l|}{ Rurality } \\
\hline Non-metro (adjacent to urban area) & $\begin{array}{l}-0.0091 * \\
(-0.0051)\end{array}$ & $\begin{array}{l}-0.0077 \\
(-0.0053)\end{array}$ & $\begin{array}{l}-0.0015 \\
(-0.0052)\end{array}$ & $\begin{array}{l}-0.022 * * \\
(-0.0051)\end{array}$ \\
\hline Non-metro (non-adjacent to urban area) & $\begin{array}{l}-0.055 * * \\
(-0.0084)\end{array}$ & $\begin{array}{l}-0.046 * * \\
(-0.0085)\end{array}$ & $\begin{array}{l}-0.034 * * \\
(-0.0081)\end{array}$ & $\begin{array}{l}-0.077 * * \\
(-0.0078)\end{array}$ \\
\hline Enrollee age & $\begin{array}{l}-0.0018 * * \\
(-0.00019)\end{array}$ & $\begin{array}{l}-0.0034 * * \\
(-0.00021)\end{array}$ & $\begin{array}{l}2.433-05 \\
(-0.00021)\end{array}$ & $\begin{array}{l}-0.0023 * * \\
(-0.00020)\end{array}$ \\
\hline Female Enrollee & $\begin{array}{l}0.0045 \\
(-0.0042)\end{array}$ & $\begin{array}{l}-0.00031 \\
(-0.0044)\end{array}$ & $\begin{array}{l}0.017 * * \\
(-0.0043)\end{array}$ & $\begin{array}{l}-0.0073^{*} \\
(-0.0042)\end{array}$ \\
\hline Months of Medicaid eligibility & $\begin{array}{l}0.024 * * \\
(-0.00088)\end{array}$ & $\begin{array}{l}0.019 * * \\
(-0.00088)\end{array}$ & $\begin{array}{l}0.014^{* *} \\
(-0.0010)\end{array}$ & $\begin{array}{l}0.010 * * \\
(-0.0010)\end{array}$ \\
\hline FQHC & $\begin{array}{l}0.027 * * \\
(-0.0061)\end{array}$ & $\begin{array}{l}0.038 * * \\
(-0.0062)\end{array}$ & $\begin{array}{l}0.041 * * \\
(-0.0064)\end{array}$ & $\begin{array}{l}0.052 * * \\
(-0.0064)\end{array}$ \\
\hline $\mathrm{RHC}$ & $\begin{array}{l}0.067 * * \\
(-0.0078)\end{array}$ & $\begin{array}{l}0.080 * * \\
(-0.0079)\end{array}$ & $\begin{array}{l}0.14 * * \\
(-0.0086)\end{array}$ & $\begin{array}{l}0.0063 \\
(-0.008)\end{array}$ \\
\hline Female provider & $\begin{array}{l}0.063 * * \\
(-0.0044)\end{array}$ & $\begin{array}{l}0.087 * * \\
(-0.0045)\end{array}$ & $\begin{array}{l}0.034 * * \\
(-0.0047)\end{array}$ & $\begin{array}{l}0.067 * * \\
(-0.0047)\end{array}$ \\
\hline County percent under poverty & $\begin{array}{l}0.0014 * \\
(-0.00059)\end{array}$ & $\begin{array}{l}-0.0027 * * \\
(-0.00061)\end{array}$ & $\begin{array}{l}0.0029 * * \\
(-0.00058)\end{array}$ & $\begin{array}{l}-0.00017 \\
(-0.00058)\end{array}$ \\
\hline County median income & $\begin{array}{l}8.46 \mathrm{e}-07 * \\
(-4.06 \mathrm{e}-07)\end{array}$ & $\begin{array}{l}-1.36 \mathrm{e}-06^{* *} \\
(-4.19 \mathrm{e}-07)\end{array}$ & $\begin{array}{l}-1.10 \mathrm{e}-06^{* *} \\
(-4.16 \mathrm{e}-07)\end{array}$ & $\begin{array}{l}-5.05 \mathrm{e}-07 \\
(-4.03 \mathrm{e}-07)\end{array}$ \\
\hline County no. of psychiatrists & $\begin{array}{l}-8.27 \mathrm{e}-05 * * \\
(-2.46 \mathrm{e}-05)\end{array}$ & $\begin{array}{l}-1.24 \mathrm{e}-05 \\
(-2.68 \mathrm{e}-05)\end{array}$ & $\begin{array}{l}-5.76 \mathrm{e}-05^{*} \\
(-2.46 \mathrm{e}-05)\end{array}$ & $\begin{array}{l}6.30 \mathrm{e}-05 * * \\
(-2.36 \mathrm{e}-05)\end{array}$ \\
\hline \multicolumn{5}{|l|}{ Mental health HPSA } \\
\hline Whole county & $\begin{array}{l}0.028 * * \\
(-0.0053)\end{array}$ & $\begin{array}{l}0.0038 \\
(-0.0055)\end{array}$ & $\begin{array}{l}-0.026^{* *} \\
(-0.0055)\end{array}$ & $\begin{array}{l}-0.0040 \\
(-0.0054)\end{array}$ \\
\hline Partial county & $\begin{array}{l}0.0045 \\
(-0.0047)\end{array}$ & $\begin{array}{l}0.0089 * \\
(-0.0051)\end{array}$ & $\begin{array}{l}-0.020 * * \\
(-0.0051)\end{array}$ & $\begin{array}{l}0.0075 \\
(-0.00493)\end{array}$ \\
\hline \multicolumn{5}{|l|}{ State $($ referent state $=$ Georgia $)$} \\
\hline North Carolina & $\begin{array}{l}0.40 * * \\
(-0.0047)\end{array}$ & $\begin{array}{l}0.14 * * \\
(-0.0052)\end{array}$ & $\begin{array}{l}0.080 * * \\
(-0.0048)\end{array}$ & $\begin{array}{l}0.055 * * \\
(-0.0047)\end{array}$ \\
\hline Texas & $\begin{array}{l}0.51 * * \\
(-0.0057)\end{array}$ & $\begin{array}{l}0.20 * * \\
(-0.0061)\end{array}$ & $\begin{array}{l}0.12 * * \\
(-0.0060)\end{array}$ & $\begin{array}{l}0.10 * * \\
(-0.0060)\end{array}$ \\
\hline \multicolumn{5}{|l|}{ Year $($ referent year $=2009)$} \\
\hline 2010 & $\begin{array}{l}-0.017 * * \\
(-0.0042)\end{array}$ & $\begin{array}{l}-0.0040 \\
(-0.0043)\end{array}$ & $\begin{array}{l}0.011 * * \\
(-0.0044)\end{array}$ & $\begin{array}{l}-0.0038 \\
(-0.0042)\end{array}$ \\
\hline 2011 & $\begin{array}{l}-0.070 * * \\
(-0.0048)\end{array}$ & $\begin{array}{l}-0.053 * * \\
(-0.0050)\end{array}$ & $\begin{array}{l}-0.11 * * \\
(-0.0048)\end{array}$ & $\begin{array}{l}-0.025 * * \\
(-0.0048)\end{array}$ \\
\hline Pearson residuals & $\begin{array}{l}-0.0048 * \\
(-0.0027)\end{array}$ & $\begin{array}{l}-0.0066^{*} \\
(-0.0035)\end{array}$ & $\begin{array}{l}0.015 * * \\
(-0.0048)\end{array}$ & $\begin{array}{l}0.0047 * \\
(-0.00246)\end{array}$ \\
\hline
\end{tabular}

Bootstrapped standard errors in parentheses

$* * p<0.01$, * $p<0.05$

management on disparities in care," and "using information on the population served by the practice to assess equity of access." ${ }^{, 53}$ Our findings provide a baseline for future research to assess whether newer versions of the NCQA PCMH standards are more effective at reducing racial disparities in quality.

\section{Limitations}

This study has several limitations. First, enrollee-level SES data are not available in Medicaid claims data, leading us to use county-level measures. Second, PCMH recognition may not reflect services that practices provide. Non-PCMH primary care practices may engage in many of the same activities as NCQA-recognized PCMHs without seeking recognition due to financial or other concerns. Moreover, measures of enrollee experience of care are not available in administrative data. Given that enrollee preference is a crucial aspect of the IOM definition of disparities, future research should take enrollee preference into account, perhaps using survey data. Third, the three states in our study have high rates of Medicaid enrollment and located in the southern U.S. However, research has suggested that geographic region or state alone does not explain disparities in health outcomes. ${ }^{54}$ Fourth, the sample size for Latinos in the treated group is limited; however, we do see a statistically significant result in that population which indicates it is adequately powered for at least some analyses. Finally, because MAX data availability lags several years, our study (2008-2011) corresponds with Version 1 of the NCQA PCMH requirements. During the study period, North Carolina had a statewide, public Medicaid PCMH program 
Table 6 Comparison of Predicted Probabilities and Disparities Changes for Diabetes Outcomes

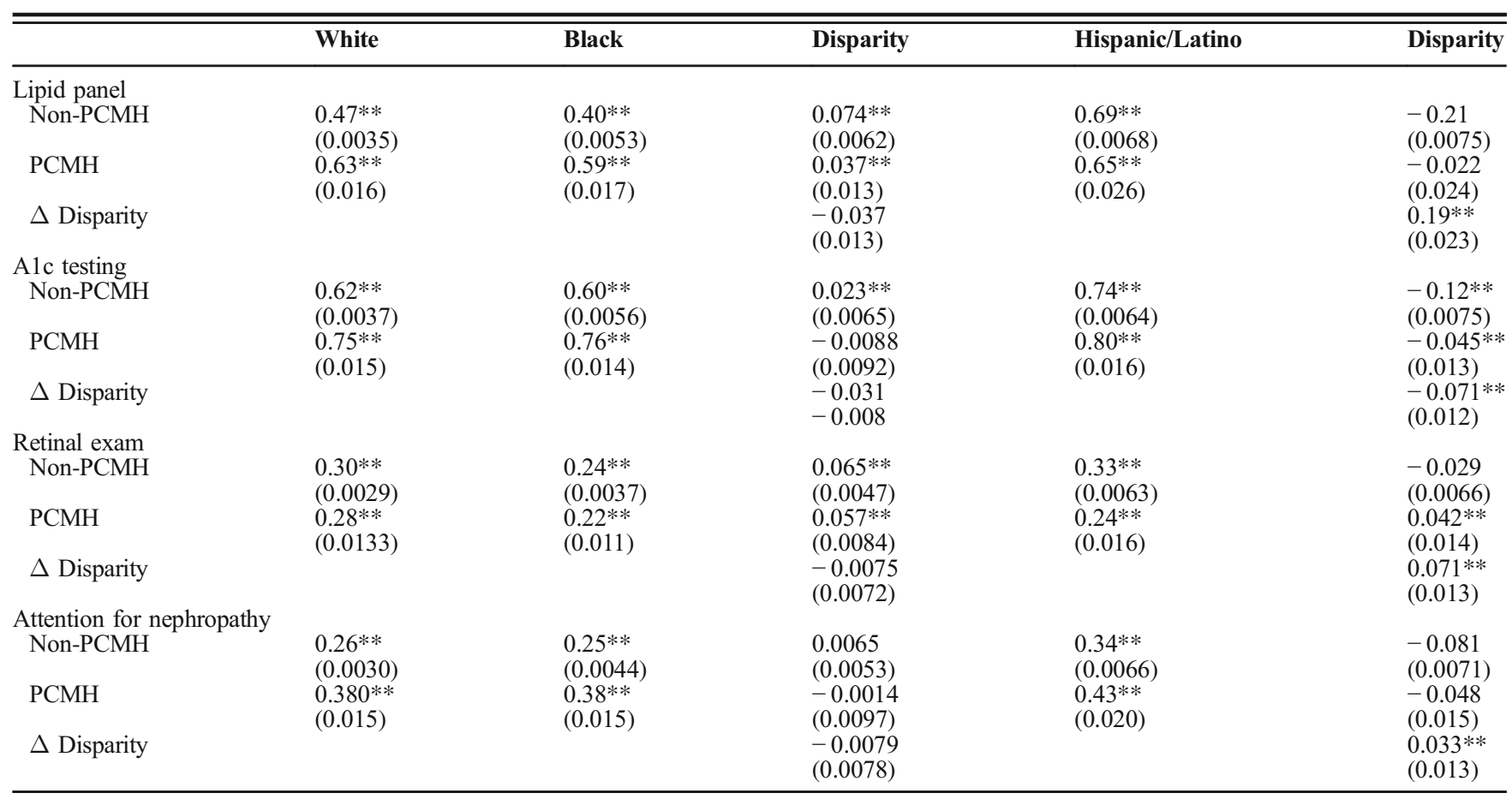

Bootstrapped standard errors in parentheses

$* * p<0.01, * p<0.05$

that predated NCQA's PCMH recognition and used different standards; Georgia and Texas have since implemented public programs incentivizing PCMHs for their Medicaid populations. ${ }^{55-58}$

\section{CONCLUSIONS}

These findings show that while the PCMH model may be associated with improvements overall quality of care, the effect is not necessarily consistent across racial groups. $\mathrm{PCMH}$ enrollment is sometimes, but not always associated with a reduction in racial disparities in quality of care, suggesting that the PCMH has potential to reduce disparities but implementing a PCMH model alone may not be sufficient to address the factors driving disparities. Providers and policymakers should explicitly consider the unique needs of minority populations in designing, implementing, and evaluating $\mathrm{PCMH}$ programs.

Acknowledgments: A preliminary version of these results was presented as a poster at the 7th Conference of the American Society of Health Economists in June 2018.

Corresponding Author: Karen E. Swietek, PhD MPH; Department of Health Policy and Management, Gillings School of Global Public Health University of North Carolina at Chapel Hill, Chapel Hill, NC, USA (e-mail: swietek-karen@norc.org).

Funding Information This work was funded by the Agency for Healthcare Research \& Quality Grants for Health Services Research Dissertation Program [grant no. R36 HS25562-O] and supported by the National Center for Advancing Translational Sciences (NCATS), National Institutes of Health [Award Number UL1TR001111].

\section{Compliance with Ethical Standards:}

The authors declare that they do not have a conflict of interest.

Disclaimer: The content is solely the responsibility of the authors and does not necessarily represent the views of the NIH.

\section{REFERENCES}

1. Cabassa LJ, Humensky J, Druss B, et al. Do Race, Ethnicity, and Psychiatric Diagnoses Matter in the Prevalence of Multiple Chronic Medical Conditions? Med Care 2013;51(6):540-547. doi:https://doi. org/10.1097/MLR.0b013e31828dbb19

2. Parekh A, Barton M. The challenge of multiple comorbidity for the us health care system. J Am Med Assoc 2010;303(13):1303-1304. doi:https://doi.org/10.1001/jama.2010.381

3. Vogeli C, Shields AE, Lee TA, et al. Multiple chronic conditions: prevalence, health consequences, and implications for quality, care management, and costs. J Gen Intern Med 2007;22 Suppl 3:391-395. doi:https://doi.org/10.1007/s11606-007-0322-1

4. Druss BG, Zhao L, Cummings JR, Shim RS, Rust GS, Marcus SC. Mental comorbidity and quality of diabetes care under medicaid: A 50state analysis. Med Care 2012;50(5):428-433. doi:https://doi.org/10. 1097/MLR.0b013e318245a528

5. Blount A, Ed D. Integrated Primary Care : Organizing the Evidence By. Fam Syst Heal 2003;21:121-134.

6. Anderson G. Chronic Care: Making the Case for Ongoing Care. Princeton, NJ; 2010. http://www.rwjf.org/content/dam/farm/reports/ reports/2010/rwjf54583. Accessed February 12, 2016.

7. Watkins DC, Assari S, Johnson-Lawrence V. Race and Ethnic Group Differences in Comorbid Major Depressive Disorder, Generalized Anxiety Disorder, and Chronic Medical Conditions. J Racial Ethn Heal Disparities 2015;2(3):385-394. doi:https://doi.org/10.1007/s40615-015-0085-Z

8. Chapa T. Mental Health Services in Primary Care Settings for Racial and Ethnic Minority Populations. Rockville, MD; 2004. 
9. Agyemang AA, Mezuk B, Perrin P, Rybarczyk B. Quality of depression treatment in Black Americans with major depression and comorbid medical illness. Gen Hosp Psychiatry 2014;36(4):431-436. doi:https:// doi.org/10.1016/j.genhosppsych.2014.02.011

10. Stecker T, Fortney J, Steffick D, Prajapati S. The Triple Threat for Chronic Disease: Obesity, Race, and Depression. Psychosomatics. 2006;47(December):513-518.

11. Alexander L, Behavioral A, Consulting H, Druss B. Behavioral Health Homes for People with Mental Health \& Substance Use Conditions: The Core Clinical Features. Washington, DC; 2012. www.integration.samhsa. gov. Accessed January 14, 2015

12. Wagner EH. Meeting the needs of chronically ill people. BMJ. 2001;323(7319):945-946. http://www.pubmedcentral.nih.gov/articlerender.fcgi?artid=1121493\&tool=pmcentrez\&rendertype=abstract. Accessed March 25, 2015.

13. Jackson GL, Powers BJ, Chatterjee R, et al. Improving patient care. The patient centered medical home. A Systematic Review. Ann Intern Med 2013;158(3):169-178. http://www.ncbi.nlm.nih.gov/pubmed/ 24779044. Accessed May 21, 2015.

14. Fillmore H, DuBard CA, Ritter GA, Jackson CT. Health care savings with the patient-centered medical home: Community Care of North Carolina's experience. Popul Health Manag 2014;17(3):141-148. doi:https://doi.org/10.1089/pop.2013.0055

15. Scholle SH, Saunders RC, Tirodkar MA, Torda P, Pawlson LG Patient-centered medical homes in the United States. J Ambul Care Manage 2011;34(1):20-32. doi:https://doi.org/10.1097/JAC. Ob013e3181ff7080

16. Akinci F, Patel PM. Quality improvement in healthcare delivery utilizing the patient-centered medical home model. Hosp Top 2014;92(4):96-104. doi:https://doi.org/10.1080/00185868.2014.968493

17. Bitton A, Martin C, Landon BE. A Nationwide Survey of Patient Centered Medical Home Demonstration Projects. J Gen Intern Med 2010;25(6):584-592. doi:https://doi.org/10.1007/s11606-010-1262-8

18. Statements of Support | Patient-Centered Primary Care Collaborative. https://www.pcpcc.org/content/statements-support. Published 2018 Accessed February 2, 2018.

19. Tarraf W, Jensen G, González HM, Gonzalez H. Patient Centered Medical Home Care Among Near-Old and Older Race/Ethnic Minorities in the US: Findings from the Medical Expenditures Panel Survey. J Immigr Minor Health 2016;19(6):1271-1280. doi:https://doi.org/10. 1007/s10903-016-0491-2

20. Takach M. About half of the states are implementing patient-centered medical homes for their Medicaid populations. Health Aff (Millwood). 2012;31(11):2432-2440. doi:https://doi.org/10.1377/hlthaff.2012.0447

21. The Henry J. Kaiser Family Foundation. States that Reported Patien Centered Medical Homes In Place. https://www.kff.org/medicaid/stateindicator/states-that-reported-patient-centered-medical-homes-inplace $/$ ?currentTimeframe $=0 \&$ sortModel $=\% 7 \mathrm{~B} \% 22$ colId $\% 22: \% 22 \mathrm{Loca}$ tion\%22,\%22sort\%22:\%22asc\%22\%7D. Published 2018. Accessed July $28,2019$.

22. Reibling $\mathbf{N}$. The Patient-Centered Medical Home: How Is It Related to Quality and Equity Among the General Adult Population? Med Care Res Rev 2016;73(5):606-623. doi:https://doi.org/10.1177/ 1077558715622913

23. Reibling $\mathbf{N}$, Rosenthal MB. The (Missed) Potential of the Patient-centered Medical Home for Disparities. Med Care 2016;54(1):9-16. doi:https://doi. org/10.1097/MLR.0000000000000451

24. Homer CJ. Health disparities and the primary care medical home: could it be that simple? Acad Pediatr 2009;9(4):203-205. doi:https://doi.org/ 10.1016/j.acap.2009.06.001

25. Beal AC, Doty MM, Hernandez SE, Shea KK, Davis K. Closing the Divide: How Medical Homes Promote Equity in Health Care.; 2007. http://www.commonwealthfund.org/ /media/files/publications/fundreport/2007/jun/closing-the-divide\%2D\%2Dhow-medical-homes-promote-equity-in-health-care $\% 2 \mathrm{D} \% 2 \mathrm{Dresults}$-from-the-commonwealth-f/ 1035 beal_closing_divide medical_homes-pdf.pdf. Accessed 2 Mar 2018.

26. Beal A, Hernandez S, Doty M. Latino access to the patient-centered medical home. J Gen Intern Med 2009;24(3 SUPPL):514-521. doi:https://doi.org/10.1007/s11606-009-1119-1

27. Berenson J, Doty MMMM, Abrams MK, Shih A. Achieving better quality of care for low-income populations: the roles of health insurance and the medical home in reducing health inequities. Issue $\mathrm{Br}$ (Commonw ... 2012;11:1-18. https://www.ncbi.nlm.nih.gov/pubmed/22611596. Accessed 30 Dec 2017.

28. Washington DL, Steers WN, Huynh AK, et al. Racial And Ethnic Disparities Persist At Veterans Health Administration Patient-Centered
Medical Homes. Health Aff 2017;36(6):1086-1094. doi:https://doi.org/ 10.1377/hlthaff.2017.0029

29. Aysola J, Bitton A, Zaslavsky AM, Ayanian JZ. Quality and equity of primary care with patient-centered medical homes: results from a national survey. Med Care 2013;51(1):68-77. doi:https://doi.org/10. 1097/MLR.0b013e318270bb0d

30. HRSA. Area Health Resource Files. Rockville, MD; 2010. https://datawarehouse.hrsa.gov/data/datadownload.aspx\#MainContent_ctlo0 gvDD_lbl_dd_topic_ttl_0. Accessed March 2, 2018.

31. U.S. Census Bureau. Small Area Income and Poverty Estimates (SAIPE). Washington, DC; 2011.

32. Katon WJ. The comorbidity of diabetes mellitus and depression. Am J Med 2008;121(11 Suppl 2):S8-15. doi:https://doi.org/10.1016/j. amjmed.2008.09.008

33. Alakeson V, Frank RG, Katz RE. Specialty care medical homes for people with severe, persistent mental disorders. Health Aff (Millwood). 2010;29(5):867-873. doi:https://doi.org/10.1377/hlthaff.2010.0080

34. Rosenthal MB, Abrams MK, Bitton A. Recommended Core Measures for Evaluating the Patient-Centered Medical Home: Cost, Utilization, and Clinical Quality. Commonw Fund Data Br 2012;12. http://www.commonwealthfund.org/Blog/2012/May/Measuring-the-Success-of-Medical-Homes-blog.aspx. Accessed 16 Feb 2018.

35. Centers for Medicare \& Medicaid Services. Core Set of Adult Health Care Quality Measures for Medicaid (Adult Core Set).; 2016.

36. NCQA. HEDIS \&amp; Quality Measurement. http://www.ncqa.org/ hedis-quality-measurement. Published 2018. Accessed October 12, 2017.

37. Busch AB, Huskamp HA, Normand S-LT, Young AS, Goldman H, Frank RG. The Impact of Parity on Major Depression Treatment Quality in the Federal Employees Health Benefits Program After Parity Implementation. Med Care 2006;44(6):506-512. doi:https://doi.org/10.1097/01.mlr. 0000215890.30756.b2

38. Katon W, Von Korff $\mathbf{M}$, Lin E, et al. Collaborative management to achieve depression treatment guidelines. J Clin Psychiatry 1997;58 Suppl 1:20-23. http://www.ncbi.nlm.nih.gov/pubmed/9054905. Accessed 12 Dec 2017.

39. Anderson B. HEDIS Antidepressant Medication Management Measures and Performance-based Measures: An Opportunity for Improvement in Depression Care. 2007;13:90-102. file://C:/Users/ karen/Downloads/A181_AndersonS98to102 (1).pdf. Accessed September 18, 2017.

40. Dowd B, Kane R, Parashuram S, Swenson T, Coulam R, Levy J. Alternative Approaches to Measuring Physician Resource Use. Rockville, MD; 2012. https://www.cms.gov/Research-Statistics-Data-and-Systems/Statistics-Trends-and-Reports/Reports /Downloads /Alt_ Approaches_Measuring_Phys_Res_Use_Report.pdf. Accessed November 7, 2016.

41. AHRQ. HCUP Chronic Condition Indicator (CCI) for ICD-9-CM. https:// www.hcup-us.ahrq.gov/toolssoftware/chronic/chronic.jsp. Accessed March 9, 2018.

42. Smedley BD, Stith AY, Nelson AR. Unequal Treatment. National Academies Press (US); 2003. doi:https://doi.org/10.17226/12875

43. Mcguire TG, Alegria M, Cook BL, Wells KB, Zaslavsky AM. Implementing the Institute of Medicine Definition of Disparities : An Application to Mental Health Care 2006:1979-2006. doi:https://doi.org/10.1111/j. 1475-6773.2006.00583.x

44. Cook BL, McGuire TG, Meara E, et al. No Title 2009;9(1):1-21. doi:https://doi.org/10.1007/s10742-008-0039-6

45. Cook BL, McGuire TG, Zaslavsky AM. Measuring racial/ethnic disparities in health care: methods and practical issues. Health Serv Res 2012;47(3 Pt 2):1232-1254. doi:https://doi.org/10.1111/j.1475-6773. 2012.01387.x

46. Hardin JW, Hilbe JM, Hardin JW, Hilbe JM. Generalized Estimating Equations: Introduction. In: Wiley StatsRef: Statistics Reference Online. Chichester, UK: John Wiley \& Sons, Ltd; 2014. doi:https://doi.org/10. 1002/9781118445112.stat06899

47. Stock JH, Yogo M, Hall A, et al. Testing for Weak Instruments in Linear IV Regression. 2002. http://www.nber.org/papers/T0284. Accessed November 4, 2016

48. Domino ME, Kilany M, Wells R, Morrissey JP. Through the Looking Glass: Estimating Effects of Medical Homes for People with Severe Mental Illness. Health Serv Res 2017;52(5):1858-1880. doi:https://doi.org/10. $1111 / 1475-6773.12585$

49. Williams JW, Jackson GL, Powers BJ, et al. Closing the quality gap: revisiting the state of the science (vol. 2: the patient-centered medical home). Evid Rep Technol Assess (Full Rep). 2012;(208.2):1-210. http:// 
www.pubmedcentral.nih.gov/articlerender.fcgi?artid=4781427\&tool=pmcentrez\&rendertype=abstract. Accessed 2 Mar 2018.

50. Alegría M, Mulvaney-Day N, Woo M, Torres M, Gao S, Oddo V. Correlates of Past-Year Mental Health Service Use Among Latinos: Results From the National Latino and Asian American Study. Am J Public Health 2007;97(1):76-83. doi:https://doi.org/10.2105/AJPH. 2006.087197

51. Cook BL, McGuire T, Miranda J. Measuring trends in mental health care disparities, 2000 2004. Psychiatr Serv 2007;58(12):1533-1540. doi:https://doi.org/10.1176/appi.ps.58.12.1533

52. Fiscella K, Franks P, Doescher MP, Saver BG. Disparities in Health Care by Race, Ethnicity, and Language Among the Insured. Med Care 2002;40(1):52-59. http://vb3lk7eb4t.search.serialssolutions.com.libproxy.lib.unc.edu/?sid=Entrez:PubMed\&id=pmid:11748426. Accessed February 25, 2018

53. NCQA. Patient-Centered Medical Home Recognition | 2017 Standards Preview.; 2017. http://www.ncqa.org/Portals/0/Programs/Recognition/ PCMH/2017 PCMH Concepts Overview.pdf?ver=2017-03-08-220342490. Accessed March 23, 2018.

54. Murray CJL, Kulkarni SC, Michaud C, et al. Eight Americas: investigating mortality disparities across races, counties, and race-counties in the United States. PLoS Med 2006;3(9):e260. doi:https://doi.org/10. 1371/journal.pmed.0030260

55. Patient-Centered Primary Care Collaborative. Texas | Patient-Centered Primary Care Collaborative. 2018. https://www.pcpcc.org/initiatives/ texas. Accessed March 12, 2018.

56. Patient-Centered Primary Care Collaborative. Georgia | Patient-Centered Primary Care Collaborative. https://www.pcpcc.org/initiatives/Georgia. Published 2018. Accessed March 12, 2018.

57. Takach M. Reinventing Medicaid: state innovations to qualify and pay for patient-centered medical homes show promising results. Health Aff (Millwood) 2011;30(7):1325-1334. doi:https://doi.org/10.1377/hlthaff. 2011.0170

58. McCarthy D, Mueller K. Community Care of North Carolina: Building Community Systems of Care Through State and Local Partnerships. Commonw Fund 2010;20(6):501-508. http://www.ncbi.nlm.nih.gov/ pubmed/21117272.

Publisher's Note Springer Nature remains neutral with regard to jurisdictional claims in published maps and institutional affiliations.
APPENDIX

Table 7 Disease-Specific Dependent Variables

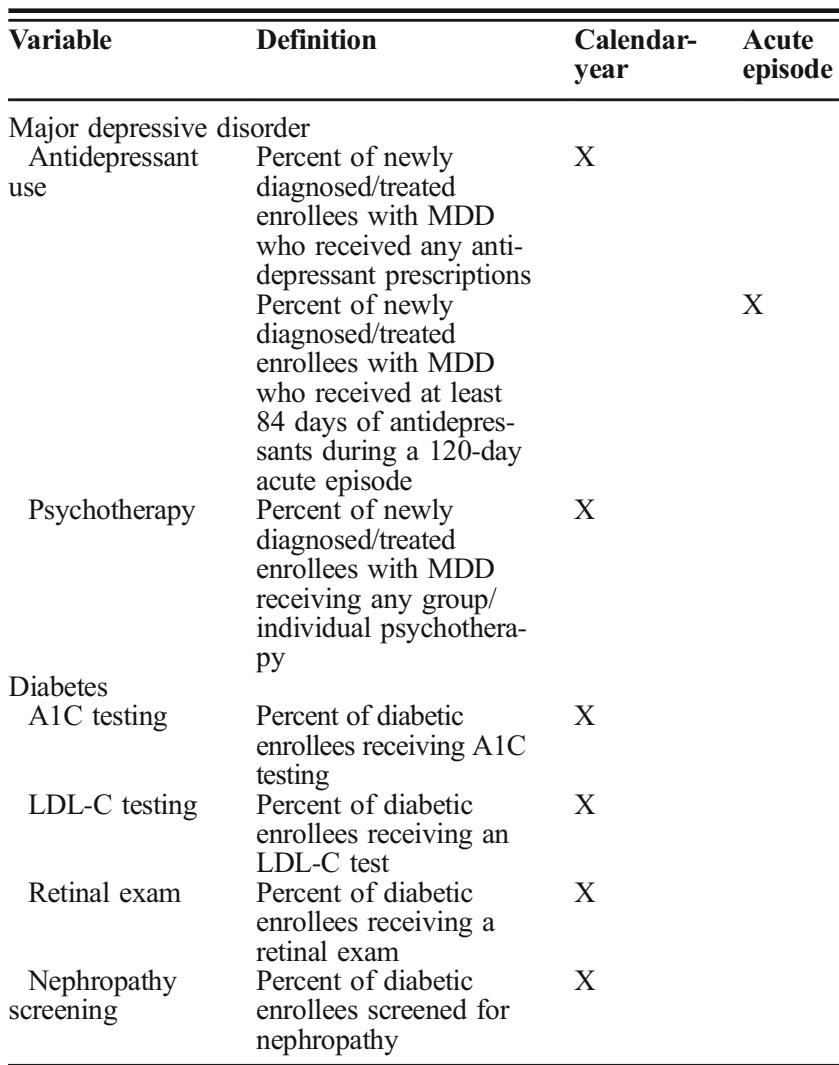

